\title{
Variations in the LISA mission
}

\author{
Bernard F Schutz \\ Max Planck Institute for Gravitational Physics (Albert Einstein Institute), Golm, Germany \\ and \\ Department of Physics and Astronomy, Cardiff University, Cardiff, UK \\ E-mail: schutz@aei.mpg.de
}

Received 5 July 2001

Published 17 September 2001

Online at stacks.iop.org/CQG/18/4145

\begin{abstract}
Before the design of LISA is frozen, it is important to consider whether changes in the design or in the mission operations would bring scientific benefits. I suggest here three possible changes: improving the shot-noiselimited sensitivity by either employing more light or larger mirrors; changing the arm-length during the mission; and inserting a fourth spacecraft into one of the arms to produce two fully independent interferometers whose noise would not be correlated. One benefit of these changes would be to significantly improve LISA's sensitivity to a cosmological background of gravitational waves. They would get LISA to within a factor of ten of the energy density upper limit of simple inflation, and they would allow LISA to verify that the window between 0.1 and $1.0 \mathrm{~Hz}$ is quiet enough to use it for exploring the cosmological background.
\end{abstract}

PACS number: $0480 \mathrm{~N}$

\section{Introduction}

The scientific goals and technical specification of LISA have evolved with time as the understanding of the technology of the mission and of its astronomical context have improved. At some point in the near future, the design of LISA will be frozen as it moves from proposal to hardware. Once LISA is launched, hopefully in 2011, it will operate for up to ten years. A LISA follow-on mission is already under informal discussion, but it might not be launched before LISA finishes its useful life, therefore sometime after 2020. It follows that we should make sure that LISA is as well matched as possible to our best current understanding of possible gravitational wave sources, and is at the same time flexible enough to change its observing programme to respond to new discoveries that may occur during its mission. The present time seems a good one at which to offer some perhaps radical suggestions for alterations in the mission, which might bring significant scientific returns. 
The desirability of any design changes at this time needs of course to be weighed against their impact on the existing mission definition, particularly if they involve added risk. Such assessments take time, and I do not attempt them here. Rather, I want only to start a discussion that could lead to a serious evaluation of the costs and benefits of suggested changes.

The thrust of the suggestions I make here is to improve LISA's sensitivity to a background of cosmological gravitational waves and increase the information from LISA that can guide a future mission dedicated to detecting a background. Many scientists, myself included, believe that the most fundamental and important observation that gravitational wave detectors could make would be to detect a random background of gravitational radiation left over from the big bang. Such a background could have been produced by a variety of non-thermal sources, such as inflation, cosmic strings, brane effects or string-type cosmologies. While the upper limits for radiation from simple inflation are low, near $\Omega_{g w} \sim 10^{-14}$ independently of frequency, some of these other processes might have created much stronger spectral features. If observed, the radiation they left behind would provide important clues - perhaps the only direct clues we will ever have - to the physics of matter at energies so high that it is and will remain simply unreachable by ground-based accelerators. For a review of this background, see Allen (1997).

Ground-based detectors will perhaps reach as low as $\Omega_{g w} \sim 10^{-11}$ when LIGO 2 begins to operate in the second half of this decade, but to go much lower than this on the ground seems unrealistic because of Earth-based gravity-gradient noise. From space, a cosmological background would probably be obscured by gravitational waves from more conventional sources over most of the standard LISA observing band from $0.1 \mathrm{mHz}$ to $0.1 \mathrm{~Hz}$. A background would have to be stronger than about $\Omega_{g w} \sim 10^{-10}$ to have a chance of being visible to the standard LISA mission. Attractive 'windows' where a background a small as $\Omega_{g w} \sim 10^{-14}$ would stand out above other gravitational wave noise are around $10^{-6} \mathrm{~Hz}$ and in the decade 0.1-1.0 Hz (Ungarelli and Vecchio 2001).

The changes in mission design suggested here are motivated primarily by the wish to get LISA closer to the inflation bound than we can expect to get from the ground, and to do it earlier than a LISA follow-on mission. It seems possible that LISA could reach a sensitivity of about $\Omega_{g w} \sim 10^{-12}$ or better in the higher-frequency window above $0.1 \mathrm{~Hz}$ if it implemented a suitable combination of the following three variations.

- Strong LISA. The shot-noise-limited sensitivity floor of the LISA noise curve could be lowered if there were more light or larger mirrors. A factor of ten in $\Omega_{g w}$ might be achievable.

- Short LISA. By shortening the arm-length by a factor of ten, LISA's sensitivity would remain optimum up to $0.1 \mathrm{~Hz}$, and it would have interesting sensitivity to $1 \mathrm{~Hz}$. The lower end of the sensitivity curve would also move up, cutting off sensitivity below $1 \mathrm{mHz}$. However, if the arm-length could be changed during the mission, then one would explore the low-frequency region during the period when the arm-length was the standard LISA baseline of $5 \times 10^{6} \mathrm{~km}$.

- Split LISA. Although for some purposes the standard LISA triangle provides two independent interferometers, they cannot simply be cross-correlated to provide an improvement in the sensitivity to a stochastic background, because the noise in the common arm exactly compensates the advantage gained from having two gravitationalwave signals. However, if a fourth spacecraft is inserted into an arm, splitting it in half, then LISA becomes two genuinely independent interferometers, and one obtains a significant improvement in cross-correlated sensitivity, particularly at higher frequencies.

Although the motivation for suggesting that these changes be examined comes from the stochastic background, there are other clear benefits to incorporating them in the mission. 
Improving the shot-noise limit improves sensitivity to the less massive black-hole coalescences, and particularly improves direction-finding for them. It might also allow us to more effectively remove some of the confusion-limited background due to compact binaries. Retaining the ability to change the arm-length during the mission gives us flexibility to adapt the mission to any new discoveries made by LISA or by other instruments during the mission. And splitting one of LISA's arms permits a more sensitive study of stellar backgrounds below $0.1 \mathrm{~Hz}$, where their spectrum and isotropy contain valuable astrophysical information.

\section{Variation 1: strong LISA}

The power spectral density $S_{h}(f)$ in the flat region between $1 \mathrm{mHz}$ and $0.1 \mathrm{~Hz}$ in the standard LISA configuration originates in laser shot noise. This can be reduced by changing the mirror diameter $d\left(S_{h} \propto d^{-4}\right)$ or the laser power $\left(S_{h} \propto P^{-1}\right)$. The sensitivity limit on $\Omega_{g w}$ is directly proportional to $S_{h}$.

Increasing the mirror diameter can be expensive, because the existing LISA mass and size budgets are tightly constrained in order to keep the launch costs down. A $30 \%$ increase to $40 \mathrm{~cm}$ diameter might be containable within the existing size and mass limits, and would increase the power $P$ and hence decrease the limit on $\Omega_{g w}$ by a factor of three (see figure 1).

The $1 \mathrm{~W}$ laser power specification has not changed since 1995. More laser power puts demands on the power supply. The solar-electric propulsion modules have larger power requirements than the payload in the present design. By integrating the modules into the payload S/C (as is proposed for short LISA in the next section), these power supplies could be made available during the mission. A more serious problem might be the optical fibres that carry the laser light to the optical system. These might not be able to handle more than $1 \mathrm{~W}$.

However, if the power can be pushed to $4 \mathrm{~W}$ and combined with $40 \mathrm{~cm}$ mirrors, this would give an 11-fold increase in sensitivity to $\Omega_{g w}$ and a 3.4-fold increase in detector range, above the binary confusion limit of $3 \mathrm{mHz}$. If the power is limited to $1 \mathrm{~W}$ but the mirrors are doubled

\section{Orientation-Averaged 1-year R.M.S Sensitivity}

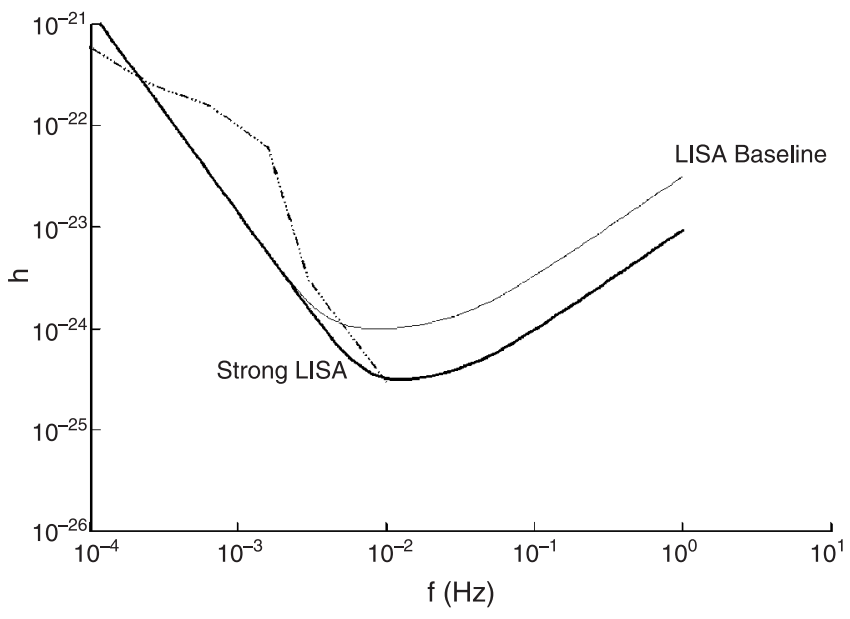

Figure 1. Strong LISA. The bold curve shows the reduction in shot-noise PSD when light increases by a factor of 11 . The baseline LISA sensitivity is shown, as is the expected confusion limit from binaries. 
in size, then the background sensitivity improvement is a factor of 16 , and the range increases by four.

\section{Variation 2: short LISA}

If LISA's spacecraft were put into a triangle whose arms were only $10 \%$ of the planned length, then essentially the LISA noise curve would move upward in frequency by a factor of ten. The lower-frequency limit would behave a little differently, but not enough to interest us here. The main reason for shortening the arms would be to gain sensitivity at higher frequencies, as shown in figure 2.

\subsection{Science at near-Hz frequencies}

There seems little attraction in making the short-arm configuration the baseline: even though there is noise from galactic binaries at low frequencies, cosmological black-hole coalescences can be seen above this noise at the lower frequencies for black holes of masses up to $10^{7} M_{\odot}$ and beyond. It is important to determine the rate of such coalescences in order to understand the way that the most massive black holes in quasars arise.

The range $0.1-1.0 \mathrm{~Hz}$ is probably a relatively quiet band (Ungarelli and Vecchio 2001), but it has not received as much theoretical attention as the main LISA band. It is one of the possible windows where an inflation-generated stochastic background might be visible above more recently generated gravitational wave noise. Few astronomical sources reach the compactness needed to generate waves of these frequencies and the higher frequencies allow one to pinpoint their direction more effectively, so that there is little chance of a confusing superposition of multiple sources. In fact, a principal purpose of exploring this frequency range would be to determine whether this window really is empty, in order to assist design of later missions.

Orientation-Averaged 1-year R.M.S Sensitivity

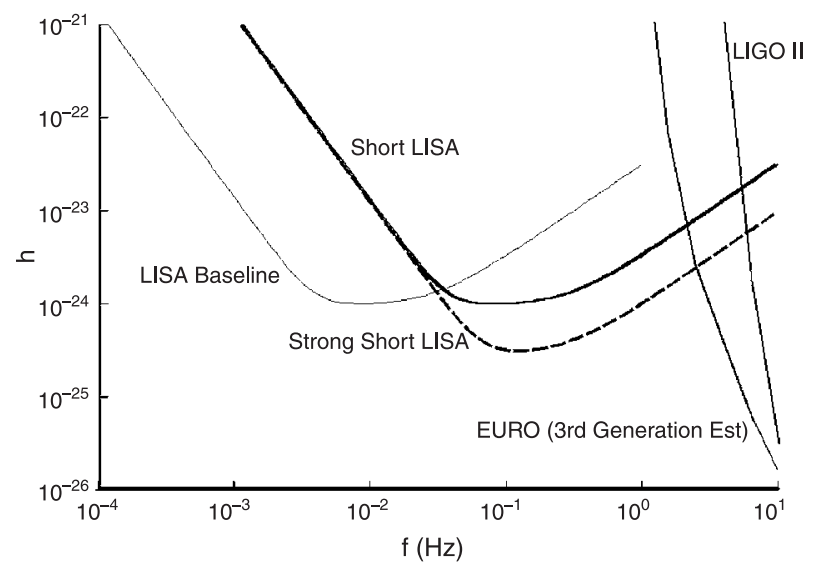

Figure 2. Short LISA. The bold curve shows how LISA's sensitivity shifts to higher frequencies when its arm-length is shortened by a factor of ten. The broken curve shows the effect of shorter arms combined with more light. For the sake of comparison, the two curves on the right show the expected LIGO 2 limit and the limit for the suggested EURO third-generation ground-based interferometer. 
Any sources seen in this frequency window would be very interesting. One possibility would be low- and intermediate-mass black hole binaries. The coalescence event (horizon merger) of $10^{4}$ and $10^{5} M_{\odot}$ binaries occurs in this window. If massive black holes in galaxies were formed by hierarchical mergers of smaller, thousand-solar-mass mergers, then these would show up in abundance. And if LISA observed a pair of $100 M_{\odot}$ black holes, it could predict the exact moment when ground-based detectors would observe their final coalescence only a few weeks later.

Another possible source would be merging white-dwarf binaries, which are a possible model for supernovae of type I. They would have to be relatively nearby for LISA to see them, however, probably not much beyond the local group of galaxies.

Of course, it would also be possible to see a cosmological background if we were lucky with its parameters, possibly one generated by meso-scale phenomena or in string-type cosmologies.

\subsection{Mission options}

If the mission were designed to retain the option of changing arm length at least once during the operational phase, then there would be no need to sacrifice observations at lower frequencies in order to explore the higher ones as well. The main decisions would be whether to use short arms at the start of the mission or its end, and how long to stay in the short-arm configuration.

Keeping this option open will not be easy technologically. The propulsion modules that move the spacecraft into their final positions would have to be retained, rather than being jettisoned from the LISA spacecraft as in the present baseline plan. Keeping the propulsion modules raises issues of the vibrations and gravitational gradients induced when their (liquid) fuel moves around in their tanks. These issues have not yet been addressed in the planning for LISA, because it was safer to separate the modules as soon as the spacecraft reached their destination.

Perhaps a new kind of propulsion engine, or redesigned fuel tanks, could overcome this problem. In any case, the problem is worst at the lower-frequency end, where the isolation system for the central mass is least effective. This suggests the strategy of going into a shortarm configuration first, where the low-frequency sensitivity is not crucial. Then, once the engines had been used again to move to longer arms, they could be jettisoned. Of course, this strategy has other risks, in particular the risk that the propulsion modules do not work when restarted, so that the mission never reaches its baseline arm-length. Such risks would need to be quantified before a decision could be made on this kind of strategy.

A safer strategy would be to go first into the long-arm configuration, accomplish the mission goals and then shorten the arms. This requires low-noise propulsion modules. It also delays acquiring information about the $0.1-1.0 \mathrm{~Hz}$ band until the end of the mission. Since one objective of observing in this band is to assist the planning for a LISA follow-on, this delay might not be the best approach for the science.

If low-noise propulsion modules can be designed, then it would be very attractive to ensure that they can be started and restarted repeatedly. One might observe for, say, three years in the baseline configuration, then shorten the arms for a year or two, then go back to the longer arms, or indeed change to yet a different arm-length that is suggested by recent astronomical observations. Arm-length flexibility is perhaps the only way that the LISA mission could be adapted to new discoveries. 


\subsection{Strong and short}

Figure 2 also shows the sensitivity curve if we combine the two suggestions made so far, namely to operate a shorter LISA with more light in the arms. This is attractive not least because it extends the region of good sensitivity right up to the limits of the ground-based detectors such as LIGO II and EURO (a preliminary estimate of the sensitivity of a proposed third-generation ground-based interferometer in Europe). Simultaneous observations between space- and ground-based gravitational wave observatories then becomes a possibility. The range for detecting WD-WD coalescences would extend over many more galaxies, perhaps as far as the Virgo Cluster.

\section{Variation 3: split LISA}

Figure 3 illustrates how a fourth spacecraft, with a slightly different design, could be used to break up a single arm and produce two interferometers with no common arm. The interferometers would have unbalanced arms, but with some attention to the optical configuration it may be possible to circulate the light twice up and down the short arms before performing the measurement. By cross-correlating the signal in the two interferometers, one can detect a common gravitational wave signal that is below the noise of a single interferometer.

Tinto, Armstrong and Estabrook have, at this meeting, proposed a clever method of discriminating a gravitational-wave background from an instrumental background, but this only works if the gravitational-wave background is stronger than the instrumental noise (Tinto et al 2001). It serves as a check to confirm that the excess noise really is an external signal. By placing a fourth LISA spacecraft into the configuration as proposed here, one can look for a background much weaker than the instrumental noise.

There are many possible backgrounds of stellar origin at these higher frequencies (see figure 4). The compact white-dwarf background has long been recognized as a source of confusion noise in LISA. This stops above about $0.03 \mathrm{~Hz}$, where the binaries merge. At higher

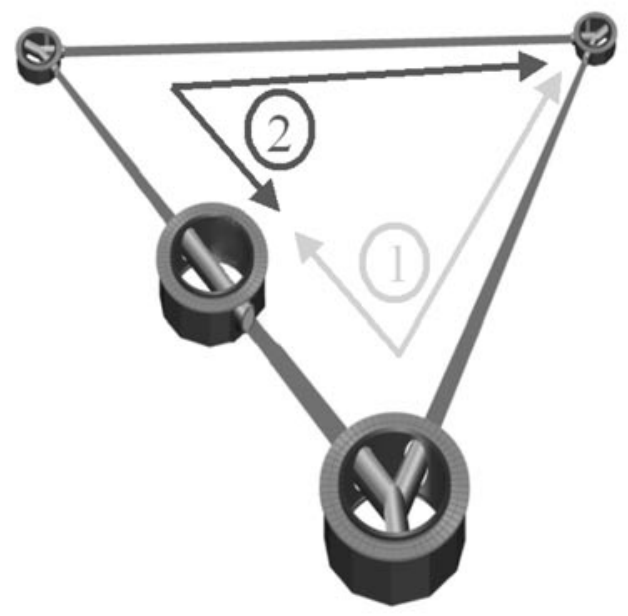

Figure 3. Split LISA. A fourth spacecraft with two telescopes anti-aligned rather than in the conventional ' $Y$ ' could be inserted into one arm to turn LISA into two fully independent interferometers with the same burst sensitivity as the original configuration but better sensitivity to a stochastic background. 


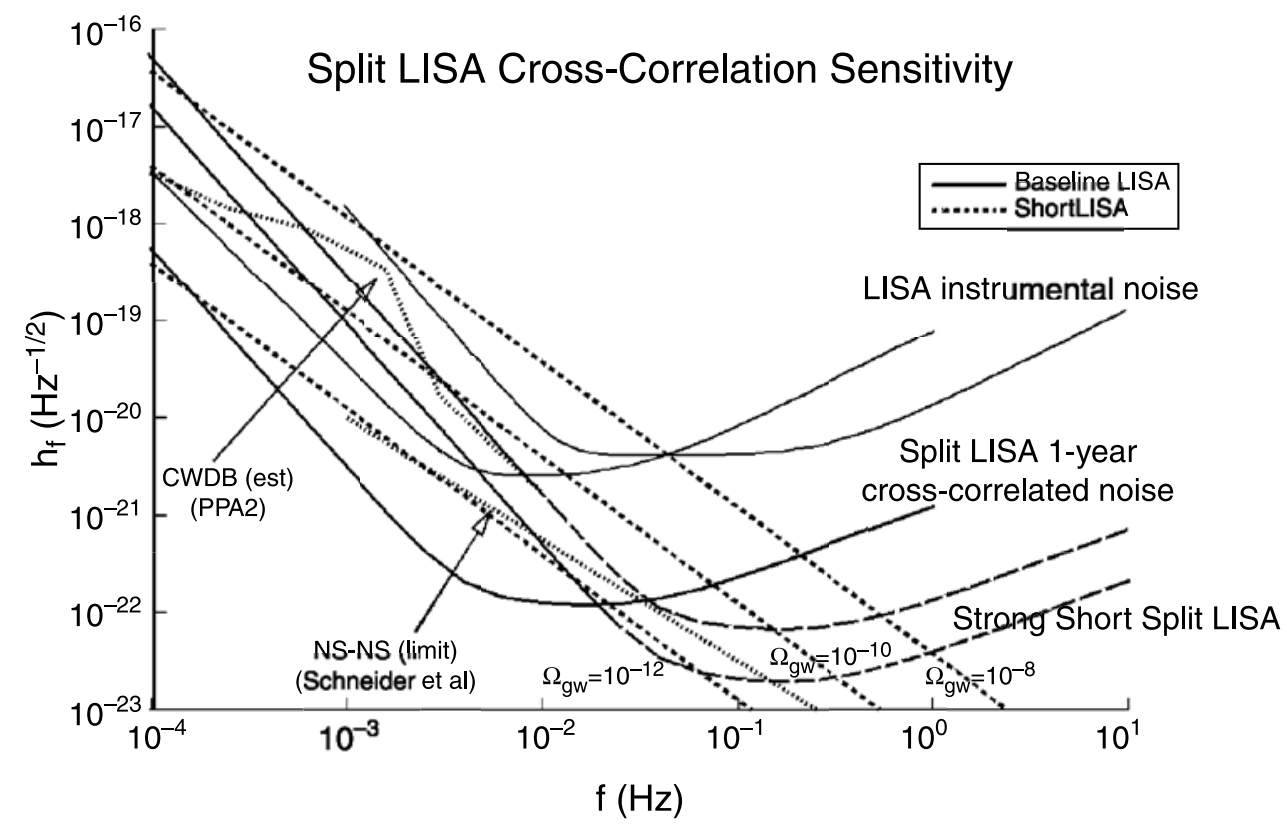

Figure 4. The various LISA configurations with the strength of a stochastic background. The background is shown for scale-free spectra down to an energy density $\Omega_{g w}=10^{-12}$. A background this small would be obscured by an extragalactic neutron-star-binary background if it meets the upper limit estimated by Schneider et al. The sensitivity of the split configuration assumes an observation of one year. LISA in its strong, short, split configuration could actually observe this strong a background and determine if a future mission to observe the stochastic background in this frequency range would be likely to fail.

frequencies there could be noise from NS-NS binaries, BH-BH binaries, old pulsars and MACHOs (if they are compact objects). Some have been studied recently by Schneider (2001) and by Ungarelli and Vecchio (2001). Popcorn noise from supernovae may be distinguishable from the conventional stochastic background. Interesting to measure for its implications for measuring the early initial mass function for massive star formation.

The interferometry in a split LISA configuration is not straightforward. It needs to be studied to see whether it is feasible or even possible. A clear problem is that spacecraft at the ends of the split arm see two lasers, one from the central spacecraft and one from the far end. Perhaps there is a solution using modulation and/or polarization. Moreover, one would have to check that signal extraction is still possible once all the controls have been applied. On the other hand, the risks associated with splitting an arm are minimal: if something goes wrong the central spacecraft can simply be turned off and the original LISA configuration can be re-acquired.

\section{Conclusion}

The point of this paper has been to offer three different modifications of the LISA baseline that each brings advantages and risks. The choice of doing one or more depends on a careful cost/benefit and feasibility analysis, which has not been done. Until that is done, it is interesting to speculate on how a mission might include them all. 
- Begin the mission with an enhanced light system, with a ten-times improvement in baseline sensitivity.

- Spend the first three years in the baseline configuration.

- Then insert a fourth spacecraft into the configuration and begin a correlation experiment for one year. This delay would allow the space agencies to wait to see successful operation of the baseline mission before committing themselves to the launch of the fourth spacecraft, and it allows a stochastic search early enough to assist planning for a LISA follow-on.

- Next shorten the arms and observe in split mode for a further year, again allowing the exploration of the higher-frequency band in time to affect planning for a LISA follow-on.

- Finally, re-adjust the arm-length according to what has been observed so far, probably back to the baseline length for five more years of black-hole observations.

Such a mission would have the flexibility to respond to its own observations and those of other astronomers, or indeed to theoretical predictions by high-energy physicists. This flexibility needs to be treated carefully, however, and it is important that planning for it be incorporated into the LISA mission design as soon as possible.

\section{References}

Allen B 1997 Proc. Les Houches School on Astrophysical Sources of Gravitational Waves ed J A Marck and J P Lasota (Cambridge: Cambridge University Press) p 373

Schneider R 2001 Class. Quantum Grav. 18

Tinto M, Armstrong J W and Estabrook F 2001 Class. Quantum Grav. 18

Ungarelli C and Vecchio A 2001 Phys. Rev. D 63064030 